\title{
UNIFORMLY PERFECT SETS AND DISTORTION OF HOLOMORPHIC FUNCTIONS
}

\author{
JIAN-HUA ZHENG ${ }^{1}$
}

\begin{abstract}
We investigate the uniform perfectness on a boundary point of a hyperbolic open set and distortion of a holomorphic function from the unit disk $\Delta$ into a hyperbolic domain with a uniformly perfect boundary point, especially of a universal covering map of such a domain from $\Delta$, and we obtain similar results to celebrated Koebe's Theorems on univalent functions.
\end{abstract}

\section{$\S 1$. Uniformly perfect points}

We begin by recalling the basic knowledge of the hyperbolic metric on a hyperbolic domain $\Omega$ in the complex plane $\mathbf{C}$, that is, $\mathbf{C} \backslash \Omega$ contains at least two points. On an arbitrary hyperbolic domain $\Omega$, we have the hyperbolic metric $\lambda_{\Omega}(z)|d z|$ with Gaussian curvature -4 . The hyperbolic metrics of the unit disk $\Delta$ and the upper half plane $\mathbf{H}=\{\operatorname{Im} z>0\}$ are respectively

$$
\lambda_{\Delta}(z)|d z|=\frac{|d z|}{1-|z|^{2}} \text { and } \lambda_{\mathbf{H}}(z)|d z|=\frac{|d z|}{2 \operatorname{Im} z} .
$$

The density $\lambda_{\Omega}(w)$ of the hyperbolic metric on a hyperbolic domain $\Omega$ is then defined as follows. Let $f(z)$ be a holomorphic universal covering map from $\Delta$ onto $\Omega$. Then the density $\lambda_{\Omega}(w)$ is determined by

$$
\lambda_{\Omega}(f(z))\left|f^{\prime}(z)\right|=\frac{1}{1-|z|^{2}} .
$$

Noting that $f(z)$ is locally homeomorphic, we can solve $\lambda_{\Omega}(w)$ from equation (1). The determination of $\lambda_{\Omega}$ is independent of the choices of holomorphic covering maps of $\Omega$ from $\Delta$ because of invariance of the hyperbolic metric $|d z| /\left(1-|z|^{2}\right)$ under Möbius transformation from $\Delta$ onto itself. Then the

Received March 23, 1999.

Revised July 17, 2000.

2000 Mathematics Subject Classification: Primary 30C80, Secondary 30F45.

${ }^{1}$ This work is supported by NSF of China and BRF of Tsinghua University. 
hyperbolic metric is conformally invariant. By $\lambda_{0,1}(z)$ we denote the density of the hyperbolic metric on $\mathbf{C} \backslash\{0,1\}$. From [8] and [9], we have

$$
\lambda_{0,1}(z) \geq \frac{1}{2|z|(|\log | z||+\kappa)},
$$

where $\kappa=\Gamma(1 / 4)^{4} /\left(4 \pi^{2}\right)$. Next, by $\bmod (A)$ we denote the modulus of an annulus $A$. Let $A=\{z ; r<|z-a|<R\}, 0<r<R$. A calculation implies that whenever $|z-a|=\sqrt{r R}$, we have

$$
\lambda_{A}(z)=\frac{\pi}{2 \sqrt{r R} \bmod (A)}
$$

(see $[4])$.

Throughout, let $W$ be a hyperbolic open set in the complex plane, that is, $\mathbf{C} \backslash W$ is closed and contains at least two points. We can define the hyperbolic metric on $W$ as the hyperbolic metric on each connected component of $W$. By $\lambda_{W}(z)$ we denote the density of the hyperbolic metric on $W$. For $a \notin W \cup\{\infty\}$, put

$$
C(a, W):=\inf \left\{\lambda_{W}(z)|z-a| ; z \in W\right\} .
$$

If $C(a, W)>0$, then $a$ is called a uniformly perfect point with respect to $W$.

For any $z_{0} \in W$, put $c\left(z_{0}, W\right):=\lambda_{W}\left(z_{0}\right) \delta_{W}\left(z_{0}\right)$, where $\delta_{W}\left(z_{0}\right):=$ $\operatorname{dist}\left(z_{0}, \partial W\right)$ throughout denotes the euclidean distance from $z_{0}$ to $\partial W$. Then

$$
\left\{z ;\left|z-z_{0}\right|<\frac{c\left(z_{0}, W\right)}{\lambda_{W}\left(z_{0}\right)}\right\} \subset W .
$$

Now we introduce a domain constant

$$
C_{W}:=\inf \{c(z, W) ; z \in W\} .
$$

In general, $0 \leq C_{W} \leq \frac{1}{2}$ (see [7]). If every component of $W$ is simply connected, from Koebe $\frac{1}{4}$ Theorem, we easily prove $\frac{1}{4} \leq C_{W}$. And $C_{W}=\frac{1}{2}$ if and only if every component of $W$ is convex (see [7]). $\partial W$ is called uniformly perfect, provided that $C_{W}>0$. There exist many mutually equivalent conditions of uniform perfectness of a closed set (see [19] and [7]).

Proposition 1. $C_{W}=\inf _{a \in \partial W \backslash\{\infty\}}\{C(a, W)\}$. 
Proof. Obviously, for any $a \in \partial W \backslash\{\infty\}, C(a, W) \geq C_{W}$. So we only need to prove that

$$
C_{W} \geq \inf _{a \in \partial W \backslash\{\infty\}}\{C(a, W)\}
$$

For any $n>0$, there exists a $z_{n} \in W$ such that $C_{W}+\frac{1}{n}>\lambda_{W}\left(z_{n}\right) \delta_{W}\left(z_{n}\right)$ and for $z_{n}$ we have $a_{n} \in \partial W \backslash\{\infty\}$ such that $\left|z_{n}-a_{n}\right|=\delta_{W}\left(z_{n}\right)$. Therefore,

$$
C_{W}+\frac{1}{n}>\lambda_{W}\left(z_{n}\right)\left|z_{n}-a_{n}\right| \geq C\left(a_{n}, W\right) \geq \inf _{a \in \partial W \backslash\{\infty\}}\{C(a, W)\} .
$$

From this (4) follows.

Hence when $\partial W$ is uniformly perfect, any finite point on $\partial W$ is a uniformly perfect one with respect to $W$. An annulus $A$ is said to separate $a$ from $\infty$ if the bounded component of $\mathbf{C} \backslash A$ contains $a$. Below we introduce two domain constants and a notation. For $a \notin W \cup\{\infty\}$, define

$\operatorname{Mod}_{a}^{0}(W):=\sup \{\bmod (A) ; A$ is a round annulus in $W$ centered at $a\}$,

$\operatorname{Mod}_{a}(W):=\sup \{\bmod (A) ; A$ is a (topological) annulus in $W$

$$
\text { and separates } a \text { from } \infty\} \text {, }
$$

where conventionally $\operatorname{Mod}_{a}^{0}(W)=0\left(\operatorname{Mod}_{a}(W)=0\right)$ if $W$ does not contain any round annuli centered at $a$ (any annuli which separate $a$ from $\infty$ ), and

$$
\beta_{W}(z ; a):=\inf \left\{\left|\log \frac{|z-a|}{|b-a|}\right| ; b \in \partial W\right\} .
$$

Since a round annulus in $W$ centered at $a$ obviously separates $a$ from $\infty$, we have $\operatorname{Mod}_{a}(W) \geq \operatorname{Mod}_{a}^{0}(W)$. We shall establish an inequality concerning $C(a, W)$ and $\operatorname{Mod}_{a}^{0}(W)$. To this end, we first prove the following result.

LEmma. For $a \in \partial W \backslash\{\infty\}$, we have

$$
\operatorname{Mod}_{a}^{0}(W)=2 \sup _{z \in W} \beta_{W}(z ; a) .
$$

Proof. For $z_{0} \in W$ with $\beta_{W}\left(z_{0} ; a\right) \neq 0$, it is clear that $\{|z-a|=$ $\delta\} \cap \partial W=\emptyset$, where $\delta=\left|z_{0}-a\right|$. Then there must exist in $W$ a round annulus $A=\{z ; r<|z-a|<R\}$ such that $\partial A \cap \partial W \neq \emptyset$ and $\delta=\sqrt{r R}$. For $b \in \partial A \cap \partial W$, then it is easy to see that

$$
\beta_{W}(z ; a)=|\log | \frac{z-a}{b-a}||=\frac{1}{2} \log \frac{R}{r}=\frac{1}{2} \bmod (A),
$$


whenever $|z-a|=\sqrt{r R}$, especially,

$$
2 \beta_{W}\left(z_{0} ; a\right)=\bmod (A) \leq \operatorname{Mod}_{a}^{0}(W) .
$$

This inequality still holds for $z_{0} \in W$ with $\beta_{W}\left(z_{0} ; a\right)=0$. Therefore

$$
2 \sup _{z \in W} \beta_{W}(z ; a) \leq \operatorname{Mod}_{a}^{0}(W)
$$

To get (5) we need to prove the converse inequality. We may assume that $\operatorname{Mod}_{a}^{0}(W)>0$, then there exists a sequence of round annuli

$$
A_{n}=\left\{z ; r_{n}<|z-a|<R_{n}\right\} \subset W
$$

such that $\partial A_{n} \cap \partial W \neq \emptyset$ and

$$
\bmod \left(A_{n}\right)+\frac{1}{n}>\operatorname{Mod}_{a}^{0}(W)
$$

Applying (6) to $A_{n}$ gives $2 \beta_{W}(z ; a)=\bmod \left(A_{n}\right)$ whenever $|z-a|=\sqrt{r_{n} R_{n}}$. Thus

$$
2 \sup _{z \in W} \beta_{W}(z ; a)+\frac{1}{n}>\operatorname{Mod}_{a}^{0}(W) .
$$

(5) immediately follows by combining (8) with (7).

We can prove by applying (2) and the method in [4] the following theorem, which is essentially due to Beardon and Pommerenke [4](see [20] and $[23])$.

Theorem A. For $a \in \partial W \backslash\{\infty\}$, we have

$$
\frac{1}{2\left(\beta_{W}(z ; a)+\kappa\right)} \leq \lambda_{W}(z)|z-a| \leq \frac{\pi}{4 \beta_{W}(z ; a)}, z \in W .
$$

Combining Theorem A with Lemma immediately shows the following result.

Proposition 2. For $a \in \partial W \backslash\{\infty\}$, we have

$$
\frac{1}{\operatorname{Mod}_{a}^{0}(W)+2 \kappa} \leq C(a, W) \leq \frac{\pi}{\operatorname{2Mod}_{a}^{0}(W)}
$$


Observe the domain

$$
\Omega_{0}:=\mathbf{C} \backslash \bigcup_{n=1}^{\infty}\left[r_{n}, r_{n}^{2}\right]
$$

where $r_{n}$ is chosen to satisfy $r_{n+1}>r_{n}^{3}>0$ and $r_{n} \rightarrow+\infty$. It is easy to see that $C_{\Omega_{0}}=0$ and from Proposition 2 for any $a \in \partial \Omega_{0} \backslash\{\infty\}, C\left(a, \Omega_{0}\right)=0$. Hence in order to consider the local structure of $\partial W$ at a boundary point $a$, we introduce the quantity

$$
C(a, W ; R):=\inf \left\{\lambda_{W}(z)|z-a| ; z \in W \cap\{|z-a|<R\}\right\},
$$

where $R$ is a positive constant. For a fixed $a, C(a, W ; R)$ decreases as $R$ increases, hence we easily prove that

$$
C(a, W)=\lim _{R \rightarrow+\infty} C(a, W ; R) .
$$

Then for $a \in \partial W \backslash\{\infty\}$, if $\{a\}$ is not a component of $\partial W$, it is easy to see from Proposition 2 that $C(a, W ; R)>0$. However, this condition is not necessary to $C(a, W ; R)>0$.

Set

$$
L_{W}(\gamma)=\int_{\gamma} \lambda_{W}(z)|d z|, \quad \gamma \subset W .
$$

It is the hyperbolic length of $\gamma$ on $W$. For any annulus $A$, the hyperbolic length of the core curve, denoted by $\operatorname{Core}(A)$, of $A$ is

$$
L_{A}(\operatorname{Core}(A))=\frac{\pi^{2}}{\bmod (A)} .
$$

Let $\Gamma_{W}(a)$ be the set of all the closed curves winding around $a \in \partial W \backslash\{\infty\}$ in $W$. Define for $a \in \partial W \backslash\{\infty\}$

$$
I(a, W):=\inf \left\{L_{W}(\gamma) ; \gamma \in \Gamma_{W}(a)\right\}
$$

where conventionally $I(a, W)=\infty$ if $\Gamma_{W}(a)=\emptyset$, and

$$
I_{W}:=\inf \{I(a, W) ; a \in \partial W \backslash\{\infty\}\} .
$$

Proposition 3. For $a \in \partial W \backslash\{\infty\}$, we have

$$
I(a, W) \leq \frac{\pi^{2}}{\operatorname{Mod}_{a}(W)} \leq I(a, W) \exp (I(a, W)) .
$$


Proof. For an annulus $A$ in $W$ which separates $a$ from $\infty$, we clearly have

$$
\frac{\pi^{2}}{\bmod (A)}=L_{A}(\operatorname{Core}(A)) \geq L_{W}(\operatorname{Core}(A)) \geq I(a, W),
$$

and therefore the left-hand side of (11) follows from arbitrary choice of $A$.

It remains to show the right-hand side of (11). From the definition of $I(a, W)$, there exists a sequence of closed curves $\left\{\gamma_{n}\right\}$ in $\Gamma_{W}(a)$ such that

$$
L_{W}\left(\gamma_{n}\right)<I(a, W)+\frac{1}{n}
$$

For each $n>0$, we have the geodesic $\alpha_{n}$ homotopic to $\gamma_{n}$ in $W$ such that $L_{W}\left(\gamma_{n}\right) \geq L_{W}\left(\alpha_{n}\right) . \alpha_{n} \in \Gamma_{W}(a)$ is obvious. By the collar lemma (see [14]), there exists a collar $A_{n}$ of width $\omega_{n}$ around the geodesic $\alpha_{n}$ in $W$, that is, $A_{n}=\left\{z \in W ; d_{W}\left(z, \alpha_{n}\right)<\omega_{n} / 2\right\}$, where $d_{W}\left(z, \alpha_{n}\right)$ denotes the hyperbolic distance of $z$ from $\alpha_{n}$, such that $A_{n}$ is homeomorphic to a round annulus and $\sinh \omega_{n} \sinh L_{W}\left(\alpha_{n}\right)=1$. From the proof of Theorem 5.2 and Corollary 5.3 of $[19]$ (see [13]), it follows that

$$
\frac{\pi^{2}}{\bmod \left(A_{n}\right)} \leq L_{W}\left(\alpha_{n}\right) \exp \left\{L_{W}\left(\alpha_{n}\right)\right\}
$$

so that

$$
\frac{\pi^{2}}{\operatorname{Mod}_{a}(W)} \leq\left(I(a, W)+\frac{1}{n}\right) \exp \left(I(a, W)+\frac{1}{n}\right) .
$$

This implies the right-hand side of (11).

Remark. The similar inequalities concerning $C_{\Omega}, I_{\Omega}$ and $\operatorname{Mod}(\Omega)=$ $\sup \left\{\operatorname{Mod}_{a}(\Omega) ; a \in \partial \Omega\right\}$ have been established, see [19], for a hyperbolic domain $\Omega$. From (10) and (11) we immediately have the following result.

THEOREM 1. For $a \in \partial W \backslash\{\infty\}$, the following statements are mutually equivalent.

(I) a is a uniformly perfect point with respect to $W$;

(II) $C(a, W)>0$;

(III) $I(a, W)>0$;

(IV) $\operatorname{Mod}_{a}^{0}(W)<\infty$;

(V) $\operatorname{Mod}_{a}(W)<\infty$. 
Proof. Obviously, we only need to imply (V) by (IV). Suppose that $\operatorname{Mod}_{a}(W)=\infty$, then there exists a sequence of annuli $\left\{A_{n}\right\}$ such that each $A_{n}$ separates $a$ from $\infty$ and $\bmod \left(A_{n}\right) \rightarrow \infty(n \rightarrow \infty)$, and furthermore we have a sequence of round annuli $\left\{B_{n}\right\}$ centered at $a$ such that $\bmod \left(B_{n}\right)=$ $\bmod \left(A_{n}\right)+O(1) \rightarrow \infty(n \rightarrow \infty)$. This implies $\operatorname{Mod}_{a}^{0}(W)=\infty$, which contradicts (IV).

Remark. From Theorem 1, it is easy to see that $C(a, W)=0$ if and only if there exists a sequence of annuli $\left\{A_{n}\right\}$ in $W$ such that for each $n, A_{n}$ separates $a$ from $\infty$ and $\bmod \left(A_{n}\right) \rightarrow \infty$ as $n \rightarrow \infty$. And we can also require either $\sup \left\{|z-a| ; z \in A_{n}\right\} \rightarrow 0$ or $\operatorname{dist}\left(a, A_{n}\right) \rightarrow \infty$ as $n \rightarrow \infty$.

Next, we discuss variation of the domain constant $C_{\Omega}$ of a hyperbolic domain $\Omega$ produced under a covering map. It is well-known that $C_{\Omega}$ is quasiinvariant under a conformal mapping. It was indeed proved in [12] that if $\Omega_{0}$ and $\Omega_{1}$ are conformally equivalent, then

$$
\frac{1}{B} C_{\Omega_{1}} \leq C_{\Omega_{0}} \leq B C_{\Omega_{1}},
$$

where $B=\left|1+i \operatorname{coth} \frac{\pi}{3}\right|=2.4335 \ldots$. Define

$$
\begin{aligned}
& r_{\Omega}:=\sup \left\{r ; \text { the hyperbolic } \operatorname{disk}\left\{z ; d_{\Omega}(z, q)<r\right\}\right. \text { is } \\
& \text { simply connected for all } q \in \Omega\}
\end{aligned}
$$

where $d_{\Omega}(z, q)$ throughout denotes the hyperbolic distance from $z$ to $q$ on $\Omega$. Then $I_{\Omega}=2 r_{\Omega}$ (see $\left.[11]\right)$. Let $p(z)$ be a covering map from $\Omega$ onto $p(\Omega)$. From the Principle of Hyperbolic Metric (see below Theorem B), we easily deduce $I_{\Omega} \geq I_{p(\Omega)}$, so that $r_{\Omega} \geq r_{p(\Omega)}$. Thus the same argument as in [12] can show the following

Proposition 4. Let $\Omega$ be a hyperbolic domain and $p(z)$ be a covering map from $\Omega$ onto $p(\Omega)$. Then

$$
C_{p(\Omega)} \leq B C_{\Omega}
$$

It is clear that the inequality $C_{\Omega} \leq B C_{p(\Omega)}$ does not generally hold, since an arbitrary hyperbolic domain must have a universal covering map from $\Delta$. 


\section{$\S 2$. Distortion theorems}

Distortion theorems concerning univalent analytic functions on $\Delta$ are well-known and play an important role in study of Complex Analysis. In this section, we mainly discuss distortion of holomorphic functions and universal covering maps from $\Delta$ in terms of uniform perfectness of image domains. The following is the Principle of Hyperbolic Metric (see Chapter III.3 of Nevanlinna[16] and also [15], this principle is sometimes called the SchwarzPick lemma), which is a start of our discussion in this section.

THEOREM B. Let $f(z)$ be holomorphic in $\Delta$ and $\Omega$ be a hyperbolic domain such that $f(\Delta) \subseteq \Omega$. Then

$$
\lambda_{\Omega}(f(z))\left|f^{\prime}(z)\right| \leq \lambda_{\Delta}(z), \text { for } z \in \Delta,
$$

with equality if and only if $f$ is a covering map of $\Omega$ from $\Delta$.

By applying the Principle of Hyperbolic Metric, we first of all establish a distortion theorem about a function holomorphic in $\Delta$.

THEOREM 2. Let $f(z)$ be holomorphic in $\Delta$ and $\Omega$ be a hyperbolic domain such that $f(\Delta) \subseteq \Omega$. If for some $a \in \partial \Omega \backslash\{\infty\}, c=2 C(a, \Omega)>0$, then for $z \in \Delta$ we have

$$
|f(0)-a|\left(\frac{1-|z|}{1+|z|}\right)^{1 / c} \leq|f(z)-a| \leq|f(0)-a|\left(\frac{1+|z|}{1-|z|}\right)^{1 / c}
$$

and

$$
\left|f^{\prime}(z)\right| \leq \frac{2|f(0)-a|}{c} \frac{(1+|z|)^{1 / c-1}}{(1-|z|)^{1 / c+1}}
$$

If, in addition, $C_{\Omega}>0$ and $f^{\prime}(0) \neq 0$, we have

$$
\left\{w ;|w-f(0)|<C_{\Omega}\left|f^{\prime}(0)\right|\right\} \subset \Omega .
$$

Proof. Applying the Principle of Hyperbolic Metric to $f(z)$ gives

$$
\lambda_{\Omega}(f(z))\left|f^{\prime}(z)\right| \leq \frac{1}{1-|z|^{2}}, z \in \Delta
$$

Then from the definition of $C(a, \Omega)$ we get

$$
\frac{c}{2} \frac{\left|f^{\prime}(z)\right|}{|f(z)-a|} \leq \lambda_{\Omega}(f(z))\left|f^{\prime}(z)\right| \leq \frac{1}{1-|z|^{2}} .
$$


Integrating the left-hand and right-hand sides of (17) along the segment $[0, z]$ gives

$$
c\left|\log \frac{|f(z)-a|}{|f(0)-a|}\right| \leq \log \frac{1+|z|}{1-|z|} .
$$

From this (13) follows, and by combining (17) with (13), we deduce (14).

Since $0<C_{\Omega} \leq \lambda_{\Omega}(f(0)) \delta_{\Omega}(f(0))$, from (16) we obtain

$$
C_{\Omega}\left|f^{\prime}(0)\right| \leq \delta_{\Omega}(f(0))
$$

This immediately implies (15).

Theorem 2 follows.

We remark on Theorem 2 . When $f(\Delta)$ is simply connected with $f(0)=$ 0 and $f^{\prime}(0)=1$, we have

$$
\left\{w ;|w|<\frac{1}{4}\right\} \subset f(\Delta) .
$$

This result generalizes Koebe $\frac{1}{4}$ Theorem, since we do not assume that $f(z)$ is univalent. When $f(\Delta)$ is convex with $f(0)=0$ and $f^{\prime}(0)=1$, we have $\left\{w ;|w|<\frac{1}{2}\right\} \subset f(\Delta)$.

THEOREM 3. Let $f(z)$ be a universal covering map of $\Omega$ from $\Delta$. If $d=2 C_{\Omega}>0$, then

$$
\frac{d}{2}\left|f^{\prime}(0)\right| \frac{(1-|z|)^{1 / d-1}}{(1+|z|)^{1 / d+1}} \leq\left|f^{\prime}(z)\right| \leq \frac{2}{d}\left|f^{\prime}(0)\right| \frac{(1+|z|)^{1 / d-1}}{(1-|z|)^{1 / d+1}}
$$

and

$$
|f(z)-f(0)| \leq\left|f^{\prime}(0)\right|\left\{\left(\frac{1+|z|}{1-|z|}\right)^{1 / d}-1\right\}
$$

Proof. For any $z \in \Delta$, there exists a point $a_{z} \in \partial \Omega$ such that $\delta_{\Omega}(f(z))=$ $\left|f(z)-a_{z}\right|$. From (15) it is easy to see that

$$
\left|f(0)-a_{z}\right| \geq \frac{d}{2}\left|f^{\prime}(0)\right|
$$

Noting $C\left(a_{z}, \Omega\right) \geq C_{\Omega}$ and using (13), we have

$$
\left|f(z)-a_{z}\right| \geq\left|f(0)-a_{z}\right|\left(\frac{1-|z|}{1+|z|}\right)^{1 / d} .
$$


An application of the Principle of Hyperbolic Metric yields

$$
\lambda_{\Omega}(f(z))\left|f^{\prime}(z)\right|=\frac{1}{1-|z|^{2}} .
$$

It is well-known that

$$
\lambda_{\Omega}(f(z)) \delta_{\Omega}(f(z)) \leq 1
$$

Combining the above inequalities shows

$$
\begin{aligned}
\left|f^{\prime}(z)\right| & \geq \frac{1}{1-|z|^{2}} \delta_{\Omega}(f(z)) \\
& =\frac{1}{1-|z|^{2}}\left|f(z)-a_{z}\right| \\
& \geq \frac{d}{2}\left|f^{\prime}(0)\right| \frac{(1-|z|)^{1 / d-1}}{(1+|z|)^{1 / d+1}}
\end{aligned}
$$

This is the left-hand side of (18). It is clear from (21) and (20) that

$$
\left|f(0)-a_{0}\right|=\delta_{\Omega}(f(0)) \leq \frac{1}{\lambda_{\Omega}(f(0))}=\left|f^{\prime}(0)\right| .
$$

Thus from (14) the right-hand side of (18) follows.

In order to prove (19), we note the elementary formula

$$
\int_{0}^{t} \frac{(1+x)^{\alpha-1}}{(1-x)^{\alpha+1}} d x=\frac{1}{2 \alpha}\left(\frac{1+t}{1-t}\right)^{\alpha}-\frac{1}{2 \alpha}
$$

where $\alpha$ is a non-zero real constant. For $z \in \Delta$, using the right-hand side of (18) we have

$$
|f(z)-f(0)|=\left|\int_{0}^{z} f^{\prime}(\zeta) d \zeta\right| \leq \frac{2}{d}\left|f^{\prime}(0)\right| \int_{0}^{|z|} \frac{(1+x)^{1 / d-1}}{(1-x)^{1 / d+1}} d x
$$

Thus applying (22) to the last integration on the above inequality implies (19).

Remark. (A) In Theorem 3, when $\Omega$ is simply connected, we have that $d=2 C_{\Omega} \geq 1 / 2$ and $f$ is a conformal mapping, and then it follows from (18) that

$$
\frac{1}{4}\left|f^{\prime}(0)\right| \frac{1-|z|}{(1+|z|)^{3}} \leq\left|f^{\prime}(z)\right| \leq 4\left|f^{\prime}(0)\right| \frac{1+|z|}{(1-|z|)^{3}}
$$


and from (19) that

$$
|f(z)-f(0)| \leq\left|f^{\prime}(0)\right| \frac{4|z|}{(1-|z|)^{2}} .
$$

Comparing them with the corresponding inequalities in Koebe Distortion Theorem for a conformal mapping from $\Delta$ onto $\Omega$, then we have reason to ask whether the coefficients $d / 2$ and $2 / d$ respectively in both the sides of (18) are necessary.

(B) The lower bound corresponding to (19) for $|f(z)-f(0)|$ does not exist unless $f(z)$ is conformal. This is because $f(z)$ can take $f(0)$ at other point in $\Delta$ than zero if $f(z)$ is not univalent.

Another distortion theorem on a universal covering map can be established by another way.

THEOREM 4. Let $f(z)$ be a universal covering map of $\Omega$ from $\Delta$. Assume that $d=2 C_{\Omega}>0$. Then

$$
\left|f^{\prime}(0)\right| \frac{(1-|z|)^{2 / d-1}}{(1+|z|)^{2 / d+1}} \leq\left|f^{\prime}(z)\right| \leq\left|f^{\prime}(0)\right| \frac{(1+|z|)^{2 / d-1}}{(1-|z|)^{2 / d+1}}
$$

and

$$
\left|\arg f^{\prime}(z)-\arg f^{\prime}(0)\right| \leq \frac{2}{d} \log \frac{1+|z|}{1-|z|}
$$

Proof. Let $F(z)$ be the universal covering map of $\Omega$ from $\Delta$ with $F(0)=$ 0 and $F^{\prime}(0)=1$ (Here we assume $0 \in \Omega$ for the moment). From the Principle of Hyperbolic Metric, we have

$$
\lambda_{\Omega}(F(z))\left|F^{\prime}(z)\right|=\lambda_{\Delta}(z) .
$$

Taking the logarithm of the above equality and, then, differentiating it give

$$
\frac{\partial}{\partial w}\left[\log \lambda_{\Omega}(w)\right](F(z)) F^{\prime}(z)+\frac{1}{2} \frac{F^{\prime \prime}(z)}{F^{\prime}(z)}=\frac{\partial}{\partial z} \log \lambda_{\Delta}(z)=\frac{\bar{z}}{1-|z|^{2}} .
$$

Thus

$$
\left|F^{\prime \prime}(0)\right|=2\left|\frac{\partial}{\partial w} \log \lambda_{\Omega}(0)\right|=\left|\nabla \log \lambda_{\Omega}(0)\right| .
$$

By Theorem 4 in [17] and by noting $\lambda_{\Omega}(0)=\lambda_{\Delta}(0)=1$, we have

$$
\left|\nabla \log \lambda_{\Omega}(0)\right| \leq \frac{2}{\delta_{\Omega}(0)} \leq \frac{2}{C_{\Omega}}
$$


and therefore

$$
\left|F^{\prime \prime}(0)\right| \leq \frac{4}{d}
$$

For each $z \in \Delta$ define

$$
g(\zeta):=\frac{f\left(\frac{\zeta+z}{1+\bar{z} \zeta}\right)-f(z)}{\left(1-|z|^{2}\right) f^{\prime}(z)} .
$$

It is easy to see that $g(\zeta)$ is a universal covering map from $\Delta$ onto $L(\Omega)$, where $L(w)=(w-f(z)) /\left[\left(1-|z|^{2}\right) f^{\prime}(z)\right]$ is a linear transformation. Also $g(0)=0$ and $g^{\prime}(0)=1$. A simple calculation reveals

$$
g^{\prime \prime}(0)=\left(1-|z|^{2}\right) \frac{f^{\prime \prime}(z)}{f^{\prime}(z)}-2 \bar{z} .
$$

Applying (25) to $g(\zeta)$ and noting $d=2 C_{\Omega}=2 C_{L(\Omega)}$ give

$$
\left|g^{\prime \prime}(0)\right|=\left|\left(1-|z|^{2}\right) \frac{f^{\prime \prime}(z)}{f^{\prime}(z)}-2 \bar{z}\right| \leq \frac{4}{d} .
$$

Multiply both the sides of this inequality by $|z| /\left(1-|z|^{2}\right)$ to get

$$
\left|\frac{z f^{\prime \prime}(z)}{f^{\prime}(z)}-\frac{2|z|^{2}}{1-|z|^{2}}\right| \leq \frac{4}{d} \frac{|z|}{1-|z|^{2}} .
$$

This implies

$$
\frac{2|z|^{2}-\frac{4}{d}|z|}{1-|z|^{2}} \leq \operatorname{Re} \frac{z f^{\prime \prime}(z)}{f^{\prime}(z)} \leq \frac{2|z|^{2}+\frac{4}{d}|z|}{1-|z|^{2}}
$$

and

$$
\left|\operatorname{Im} \frac{z f^{\prime \prime}(z)}{f^{\prime}(z)}\right| \leq \frac{4}{d} \frac{|z|}{1-|z|^{2}}
$$

We note

$$
\operatorname{Re} \frac{z f^{\prime \prime}(z)}{f^{\prime}(z)}=|z| \frac{\partial}{\partial|z|} \log \left|f^{\prime}(z)\right|
$$

and

$$
\operatorname{Im} \frac{z f^{\prime \prime}(z)}{f^{\prime}(z)}=|z| \frac{\partial}{\partial|z|} \arg f^{\prime}(z) .
$$

Thus (26) and (27) respectively yield

$$
\frac{2|z|-\frac{4}{d}}{1-|z|^{2}} \leq \frac{\partial}{\partial|z|} \log \left|f^{\prime}(z)\right| \leq \frac{2|z|+\frac{4}{d}}{1-|z|^{2}}
$$


and

$$
-\frac{4}{d} \frac{1}{1-|z|^{2}} \leq \frac{\partial}{\partial|z|} \arg f^{\prime}(z) \leq \frac{4}{d} \frac{1}{1-|z|^{2}} .
$$

Integrating both the sides of the above two inequalities along the segment $[0, z]$ respectively implies $(23)$ and $(24)$.

The following is a consequence of Theorems 3 and 4, which generalizes the celebrated distortion theorem of a univalent analytic function on $\Delta$.

Corollary 1. Assume that $K$ is a compact subset of hyperbolic domain $G$. Then for every covering map $f: G \rightarrow f(G)$ such that $C_{f(G)} \geq k>$ 0 , we have

$$
\frac{1}{M} \leq \frac{\left|f^{\prime}(z)\right|}{\left|f^{\prime}(w)\right|} \leq M, \text { for } z, w \in K
$$

where $M$ are a positive constant depending on $K$ and $k$.

Proof. It suffices to prove the right-hand side of (28). Let $h$ be a universal covering map of $G$ from $\Delta$. Then $g=f(h): \Delta \rightarrow f(G)$ is a covering map. We can find a $r, 0<r<1$, such that $h\left(\Delta_{r}\right) \supset K, \Delta_{r}=\{|z|<r\}$. For a pair of $z$ and $w$ in $K$, there exist $z_{0}$ and $w_{0}$ in $\Delta_{r}$ such that $h\left(z_{0}\right)=z, h\left(w_{0}\right)=w$. From Proposition 4 it follows that $s=C_{G} \geq 0.42 C_{f(G)} \geq 0.42 k>0$. Applying Theorem 4 respectively to $h$ and $g$ gives

$$
\frac{\left|h^{\prime}\left(w_{0}\right)\right|}{\left|h^{\prime}\left(z_{0}\right)\right|} \leq \frac{(1+r)^{2 / s}}{(1-r)^{2 / s}}
$$

and

$$
\frac{\left|f^{\prime}(z) h^{\prime}\left(z_{0}\right)\right|}{\left|f^{\prime}(w) h^{\prime}\left(w_{0}\right)\right|}=\frac{\left|g^{\prime}\left(z_{0}\right)\right|}{\left|g^{\prime}\left(w_{0}\right)\right|} \leq \frac{(1+r)^{2 / k}}{(1-r)^{2 / k}} .
$$

Combining the above inequalities implies the right-hand side of (28).

We can also establish the corresponding inequalities to (13) for half plane, angular domain and other special domains.

THEOREM 5. Let $f(z)$ be holomorphic in $\mathbf{H}$ and $f(\mathbf{H}) \subseteq \Omega$. If for some $a \in \partial \Omega \backslash\{\infty\}, c=2 C(a, \Omega)>0$, then for any $0<\delta<\frac{\pi}{2}$, we have

$$
|f(z)| \leq C_{0}\left(1+|z|^{1 / c}\right),\left|\arg z-\frac{\pi}{2}\right|<\delta,
$$

where $C_{0}$ is a positive constant depending on $\delta$, a and a fixed point $z_{1}$ in $\mathbf{H}$ and $f\left(z_{1}\right)$. 
Proof. It is well-known (see [3]) that for a fixed point $z_{1}$ in $\mathbf{H}$, we have

$$
\sinh ^{2} d_{\mathbf{H}}\left(z, z_{1}\right)=\frac{\left|z-z_{1}\right|^{2}}{4 \operatorname{Im}[z] \operatorname{Im}\left[z_{1}\right]}=O(|z|),
$$

whenever $\left|\arg z-\frac{\pi}{2}\right|<\delta$ and $z \rightarrow \infty$.

On the other hand, recalling the definition of hyperbolic distance between two points we obtain

$$
\begin{aligned}
d_{\Omega}\left(\zeta, \zeta_{0}\right) & =\inf _{\gamma} \int_{\gamma} \lambda_{\Omega}(\zeta)|d \zeta| \\
& \geq \frac{c}{2} \inf _{\gamma} \int_{\gamma} \frac{|d \zeta|}{|\zeta-a|} \\
& \geq \frac{c}{2}|\log | \frac{\zeta-a}{\zeta_{0}-a}||,
\end{aligned}
$$

where the infimum is taken over all the curves $\gamma$ connecting $\zeta$ and $\zeta_{0}$ in $\Omega$. Noting $\sinh ^{2} x>e^{2 x} / 4-1 / 2$, this yields

$$
\begin{aligned}
\sinh ^{2} d_{\Omega}\left(\zeta, \zeta_{0}\right) & \geq \sinh ^{2}\left\{\frac{c}{2} \log \left|\frac{\zeta-a}{\zeta_{0}-a}\right|\right\} \\
& >\frac{1}{4}\left|\frac{\zeta-a}{\zeta_{0}-a}\right|^{c}-\frac{1}{2}, \text { for } \zeta, \zeta_{0} \in \Omega .
\end{aligned}
$$

Then the desired inequality (29) can be derived from $d_{\Omega}\left(f(z), f\left(z_{1}\right)\right) \leq$ $d_{\mathbf{H}}\left(z, z_{1}\right)$ and by combining (30) with (31).

Let $D\left(z_{0}, \theta, \delta\right):=\left\{z ;\left|\arg \left(z-z_{0}\right)-\theta\right|<\delta\right\}$ be an angular domain. Transformation

$$
w=M(z)=\left\{e^{-i(\theta-\delta)}\left(z-z_{0}\right)\right\}^{\frac{\pi}{2 \delta}}
$$

maps conformally $D\left(z_{0}, \theta, \delta\right)$ onto the upper half plane $\mathbf{H}$. And $w=$ $\exp \left(\frac{\pi}{R-r}(z-R i)\right)$ maps conformally the band domain $\{r<\operatorname{Im} z<R\}$ onto the upper half plane $\mathbf{H}$. Then from Theorem 5 the following results immediately follow.

Corollary 2. Let $f(z)$ be holomorphic in $D\left(z_{0}, \theta, \delta\right)$ and $f\left(D\left(z_{0}, \theta, \delta\right)\right)$ $\subseteq \Omega$. If for some $a \in \partial \Omega \backslash\{\infty\}, c=2 C(a, \Omega)>0$, then for any $0<\delta_{0}<\delta$, we have

$$
|f(z)| \leq C_{0}\left(1+|z|^{\frac{\pi}{2 c \delta}}\right), \text { for } z \in D\left(z_{0}, \theta, \delta_{0}\right)
$$

where $C_{0}$ is a positive constant depending on $\delta_{0}, \delta$, a and a fixed point $z_{1}$ in $D\left(z_{0}, \theta, \delta_{0}\right)$ and $f\left(z_{1}\right)$. 
COROllary 3. Let $f(z)$ be holomorphic in $E=\{r<\operatorname{Im} z<R\}$ and $f(E) \subseteq \Omega$. If for some $a \in \partial \Omega \backslash\{\infty\}, c=2 C(a, \Omega)>0$, then for any $0<\delta_{0}<(R-r) / 2$, we have

$$
|f(z)| \leq C_{0} \exp \left(\frac{\pi}{(R-r) c}|z|\right), \text { for } z \in\left\{r+\delta_{0}<\operatorname{Im} z<R-\delta_{0}\right\},
$$

where $C_{0}$ is a positive constant depending on $\delta_{0}$, a and a fixed point $z_{1}$ in $E$ and $f\left(z_{1}\right)$.

Remark. The inequalities (29), (32) and (33) are sharp. For example, observe function $h(z)=\left\{e^{-i(\theta-\delta)}\left(z-z_{0}\right)\right\}^{\frac{\pi}{2 \delta}}$. It maps conformally $D\left(z_{0}, \theta, \delta\right)$ onto the upper half plane $\mathbf{H}$. Obviously, $h(z)$ satisfies the condition of Corollary 2 with $\Omega=\mathbf{H}$. In fact it is easy to see that for any $a \in\{\operatorname{Im} z=0\}$, $c=2 C(a, \mathbf{H})=1$. Thus

$$
|h(z)|=\left|z-z_{0}\right|^{\frac{\pi}{2 \delta}} \sim|z|^{\frac{\pi}{2 \delta} \frac{1}{c}}
$$

as $z \rightarrow \infty, z \in D\left(z_{0}, \theta, \delta\right)$.

Corollary 2 has an application in iteration theory of meromorphic functions. Let $f(z)$ be a transcendental meromorphic function in the complex plane. Let $f^{n}(z)$ denote the $n$-th iterate of $f: f^{1}(z)=f(z), f^{n}(z)=$ $f\left(f^{n-1}(z)\right)=f^{n-1}(f(z))$. Then $f^{n}(z)$ is defined for all $z \in \mathbf{C}$ except for a countable set of the poles of $f, f^{2}, \ldots, f^{n-1}$. Define Fatou set of $f(z)$ as $F(f):=\left\{z \in \mathbf{C} ;\left\{f^{n}\right\}\right.$ is defined and normal in some neighborhood of $\left.z\right\}$. $F(f)$ is open and each $f^{n}(z)$ is analytic in $F(f)$. It is well-known that $F(f)$ is completely invariant, that is, $z \in F(f)$ if and only if $f(z) \in F(f)$, and thus for any connected component $U$ of $F(f)$, called a stable domain of $f$, $f^{n}(U)$ is contained in a component $U_{n}$ of $F(f)$. If for some $n, U_{n}=U$, then $U$ is called a periodic domain of $f$; If for $n \neq m, U_{n} \neq U_{m}$, then $U$ is called a wandering domain of $f$. We refer to [5] for more information.

THEOREM 6. Let $f$ be a meromorphic function and $U$ be a stable domain of $f$. Assume that there exist an angular domain $D\left(z_{0}, \theta, \delta\right) \subset U$ and an $a \notin U$ such that $C(a, U)>0$. Then for any positive integer $n$, we have

$$
\left|f^{n}(z)\right| \leq C_{n}\left(1+|z|^{\frac{t \pi}{4 \delta}}\right), \text { for } z \in D\left(z_{0}, \theta, \delta_{0}\right)
$$

where $0<\delta_{0}<\delta, t=\max \{4,1 / C(a, U)\}$ and $C_{n}$ is a positive constant depending on $a, \delta_{0}, \delta, n$ and a fixed point $z_{1}$ in $U$ and $f^{n}\left(z_{1}\right)$. 
Proof. If $U_{n}=U$, then $f^{n}$ satisfies the condition of Corollary 2 with $\Omega=U$; If $U_{n} \neq U$, then $U_{n} \cap U=\emptyset$, and $U_{n} \subset \mathbf{C} \backslash\left\{\arg \left(z-z_{0}\right)=\right.$ $\theta\}$. Noting the fact that $\mathbf{C} \backslash\left\{\arg \left(z-z_{0}\right)=\theta\right\}$ is simply connected and $C_{\mathbf{C} \backslash\left\{\arg \left(z-z_{0}\right)=\theta\right\}}=1 / 4$, we also have that $f^{n}$ satisfies the condition of Corollary 2 with $\Omega=\mathbf{C} \backslash\left\{\arg \left(z-z_{0}\right)=\theta\right\}$. Thus (34) follows from Corollary 2.

We remark on Theorem 6. (I) (34) with $t=4$ holds without the assumption of $C(a, \Omega)>0$ when $U$ is a wandering domain of $f$.

(II) When $U$ is simply connected, (34) with $t=1 / C_{U} \leq 4$ holds, which was established in [6] and [18] by different methods with $t=4$ for the case when $f$ is an entire function, for an unbounded stable domain of an entire function $f$ is simply connected (see [2]).

Acknowledgements. I would like to send my many thanks to the referee for his valuable comments and suggestions in improving this paper and telling me the preprint of Kim and Sugawa[10] in which some distortion theorems were established.

\section{REFERENCES}

[1] L. Ahlfors, Conformal invariants, McGraw-Hill, New York, 1973.

[2] I.N. Baker, Wandering domains in the iteration of entire functions, Proc. London Math. Soc. (3), 49 (1984), 563-576.

[3] A.F. Beardon, The Geometry of discrete groups, Springer-Verlag, 1983.

[4] A.F. Beardon and Ch. Pommerenke, The Poincaré metric of plane domains, J. London Math. Soc. (2), 18 (1978), 475-483.

[5] W. Bergweiler, Iteration of meromorphic functions, Bull. Amer. Math. Soc., (N.S.), 29 (1993), 151-188.

[6] P. Bhattacharyya, On the domain of normality of an attractive fixpoint, Trans. Amer. Math. Soc., 135 (1971), 89-98.

[7] R. Harmelin and D. Minda, Quasi-invariant domain constants, Israel J. Math., 77 (1992), 115-127.

[8] J.A. Hempel, The Poincaré metric on the twice punctured plane and the theorems of Landau and Schottky, J. London Math. Soc., (2), 20 (1979), 435-445.

[9] J.A. Jenkins, On explicit bounds in Landau's theorem II, Canad. J. Math., 33 (1981), 559-562.

[10] Y.C. Kim and T. Sugawa, Growth and coefficient estimates for uniformly locally univalent functions on the unit disk, preprint (1999).

[11] X. Liu and D. Minda, Monotonicity of hyperbolic curvature under univalent mappings, Ann. Acad. Sci. Fenn., 16 (1991), 227-242. 
[12] W. Ma and D. Minda, Behavior of domain constants under conformal mappings, Israel J. Math., 91 (1995), 157-171.

[13] B. Maskit, Comparison of hyperbolic and extremal lengths, Ann. Acad. Sci. Fenn. Ser.A I Math., 10 (1985), 381-386.

[14] C.T. McMullen, Complex dynamics and renormalization, Princeton Univ. Press, Princeton, New Jersey, 1994.

[15] D. Minda, Inequalities of the hyperbolic metric and applications to geometric function theory, Lecture Notes in Math., Springer, Berlin-News York, 1275 (1987), 235-252.

[16] R. Nevanlinna, Analytic functions, Springer, Berlin, Heidelberg, New York, 1970.

[17] B.G. Osgood, Some properties of $f^{\prime \prime} / f^{\prime}$ and the Poincaré metric, Indiana Univ. Math. J., 31 (1982), 449-461.

[18] J.Y. Qiao, Stable sets for iteration of entire functions, Acta Math. Sinica, No.5, 37 (1994), 702-708 (in Chinese).

[19] T. Sugawa, Various domains constants related to uniform perfectness, Complex Variables, 36 (1998), 311-345.

[20] S. Yamashita, The derivative of a holomorphic function and estimates of the Poincaré density, Kodai Math. J., 15 (1992), 102-121.

[21] J.H. Zheng, Unbounded domains of normality of entire functions of small growth, Math. Proc. Cambridge Phil. Soc., 128 (2000), 355-361.

[22] - Singularities and wandering domains in iteration of meromorphic functions, Illinois J. Math., 44, no. 3, Fall (2000), 520-530.

[23] On uniformly perfect boundaries of stable domains in iteration of meromorphic functions II, to appear in Proc. Math. Cambridge Phil. Soc.

Department of Mathematical Science

Tsing Hua University

Beijing, P. R. China

jzheng@math.tsinghua. edu.cn 